\title{
Extremely rare tumour - malignant mesothelioma of tunica vaginalis testis
}

\author{
Jankovichova $\mathrm{T}^{1}$, Jankovich $\mathrm{M}^{1}$, Ondrus $\mathrm{D}^{2}$, Kajo $\mathrm{K}^{3}$, Dubravicky $\mathrm{J}^{1}$, Breza $\mathrm{J}^{1}$ \\ Department of Urology, Faculty of Medicine, Comenius University and University Hospital Bratislava, Academician's Derer \\ Hospital, Bratislava, Slovakia. visvaderovat@gmail.com
}

\begin{abstract}
INTRODUCTION: Malignant mesothelioma of tunica vaginalis testis is an extremely rare tumour. It is often caused by exposition to asbestos, however, more often its occurrence is sporadic. The diagnosis is usually set secondarily during hydrocele surgery. This type of tumour should be considered in cases with with atypical hydrocele, especially haematocele or atypical shape of seminal covering.

RESULTS: A case of an asbestos-exposed patient with described disease and long-term hydrocele is presented.

The number of patients is so small that the guidelines are limited due to low statistical power (Fig. 2, Ref. 14).

Text in PDF www.elis.sk.

KEY WORDS: mesothelioma, genital neoplasms, male; asbestos, testicular hydrocele.
\end{abstract}

\section{Introduction}

Malignant mesothelioma in any location is a rare tumour, usually caused by exposition to asbestos $(1,2)$. Malignant mesothelioma of tunica vaginalis testis is the most unusual type of mesothelioma, with only approximately 250 cases described worldwide. Due to its extremely low incidence, it is usually diagnosed secondarily during surgery for other reasons. The most common diagnosis set before the operation is hydrocele, followed by testicular tumour and inguinal hernia (3). The prognosis is usually poor with short disease-specific survival. This paper presents a case of a patient exposed to asbestos with long-term hydrocele.

\section{Case report}

A 67-year-old patient was admitted to our department for routine left hydrocele surgery. He underwent hydrocele operation three years ago in another hospital, but the hydrocele of left testis gradually developed again. Shortly before the admission to our department, he also underwent an outpatient non lege artis treatment, namely hydrocele puncture, which was not successful. Hydrocele developed again in few days. Patient's left hemiscrotum was enlarged markedly with no signs of inflammation; the skin was freely movable; palpation of the scrotum was painless

${ }^{1}$ Department of Urology, Faculty of Medicine, Comenius University in
Bratislava and University Hospital Bratislava, Academician's Derer Hos-
pital, Bratislava, Slovakia, ${ }^{2} 1$ st Department of Oncology, Faculty of Medi-
cine, Comenius University in Bratislava and St. Elisabeth Cancer Institute,
Bratislava, Slovakia, and ${ }^{3}$ Department of Pathology, Slovak Medical Uni-
versity and St. Elisabeth Cancer Institute, Bratislava, Slovakia

Address for correspondence: T. Jankovichova, Datelinova 1, SK-821 01 Bratislava, Slovakia.

Phone: +421.905688081 and revealed a solid, smooth formation in the left hemiscrotum extending to the inguinal canal. Ultrasonography revealed a considerably hyperechoic content of hydrocele with movement of hyperechoic particles during scrotal manipulation. The testis and epididymis were bilaterally without visible pathology. We considered a possibility of inguinal hernia. Therefore, the operation started with preparation of inguinal canal, which however showed no signs of inguinal herniation. After this finding, a puncture of hydrocele was performed, showing bloody content with signs of instant bleeding. Based on this, radical inguinal orchiectomy was done. Patient agreed with radical solution in case of neoplasm suspicion and signed informed consent before the operation. Detailed histopathological examination of the removed specimen showed a solid light-grey tumour with extragonadal propagation to external layers of the testis covering on the dorsal side with a pseudocystic cavern filled with fibrin on the ventral side of the testis. The spermatic cord was not affected; layers of the testicular covering were diffusely thickened and scarred. Microscopy revealed chronic fibroproliferative inflammation and bleeding into testis covering. The structure of the tumour was epithelioid, with solid-striated, partially tubular or cystopapillary proliferation. There were nests of massive bleeding and comedonecrosis, stromal hyalinisation and high mitotic activity (Figs 1 and 2). The testicle was without pathologic findings. Imunohistochemic examination showed expression of vimentin, EMA, calretinin and absence of CD 30, CD 20, and CEA. Based on the finding of malignant mesothelioma, we asked the patient about his actual and former employments and his environment. Although he was not in contact with asbestos as part of his job handling various construction materials as a lorry driver, for decades he was living in a house roofed with eternit which is a type of fibre cement used for the production of asbestos-containing roofing material (4). Patient underwent further examination; computed tomography showed absence of positive 


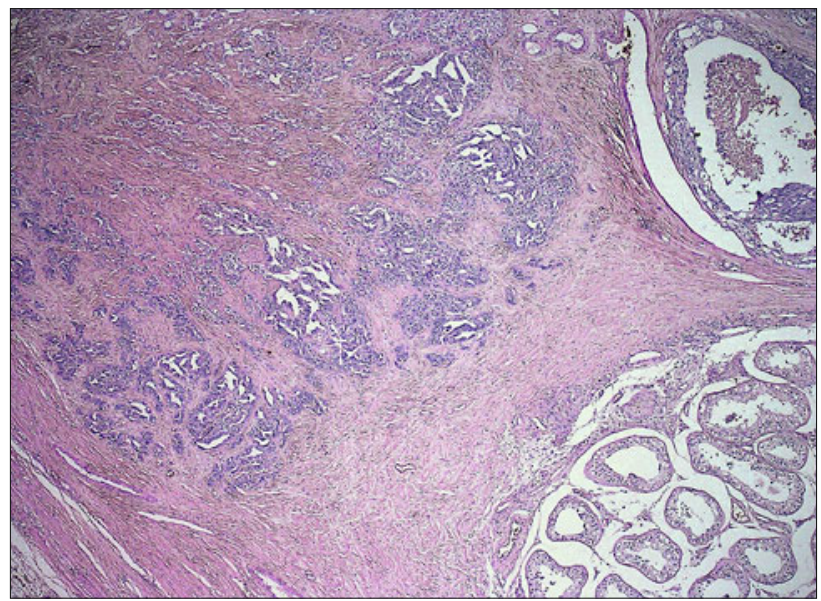

Fig. 1. Malignant mesothelioma - infiltration of tunica albuginea and tunica vaginalis with close relation to intact testicular parenchyma in lower right part (HE staining, magn. 40x).

lymph nodes or metastatic spread. Fourteen months after radical orchiectomy, the patient developed local recurrence - he presented with a painless solid mass approximately $5 \mathrm{~cm}$ in diameter on the borderline of scrotum and left inguinal area with fixed skin on the surface. Resection of the tumour was performed. Histological finding showed complete resection of tumour with negative surgical margin; imunohistochemical examination showed expression of CK 7, vimentin, CK5-6 and calretinin with absence of CD 20 and CEA. After uncomplicated operation and postoperative period patient underwent further CT scan with no signs of lymph node spread or distant metastases. Post-operative radiotherapy and chemotherapy was not indicated. Patient is 20 months after resection of local recurrence with no evidence of disease and with overall survival of 44 months after the primary treatment.

\section{Discussion}

Malignant mesothelioma of tunica vaginalis testis was first described in 1957 by Barbera and Rubino (5); since then it remains to be a very rare tumour with sporadic occurrence worldwide. Due to its extremely low incidence and prevalence, statistical evaluation of described cases would be underpowered. With only few cases worldwide, it is the most uncommon type of mesothelioma. About $50 \%$ to $70 \%$ of mesotheliomas (in any localization) are associated with exposure to asbestos (2). However, less than half of reported mesotheliomas of tunica vaginalis testis are caused by asbestos exposition. Alesawi (2013) reports only 16 cases in literature overtly exposed to asbestos compared to 42 cases with no proved exposition, and 27 unspecified cases (6), The cause could either lie in extremely small incidence, which may result in not anticipating a neoplasm and not taking precise history, or it can be also a sign that asbestos is "hidden" in our environment without our knowledge, and long-term exposure can cause this disease. Other suspected causes are scrotal trauma (7), exposition to radiation during radiotherapy (8) and long-term hydrocele (9).

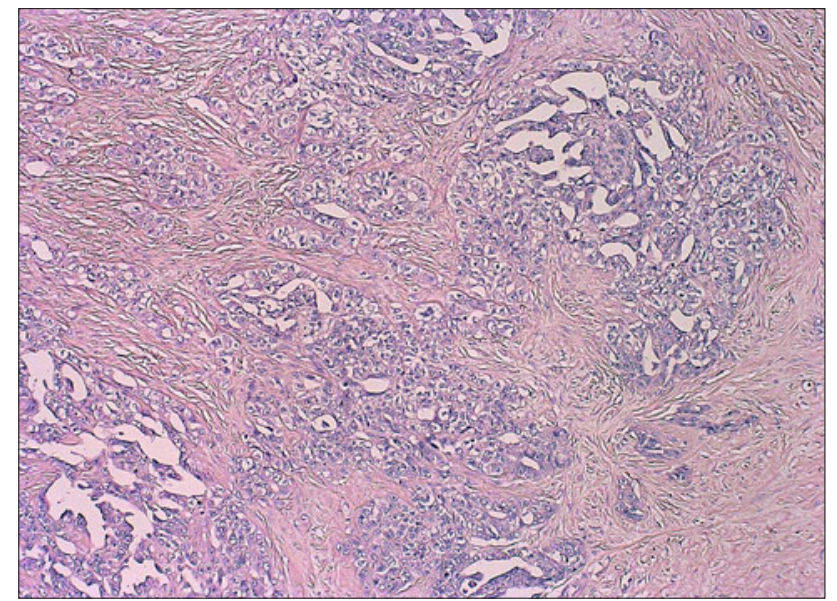

Fig. 2. Malignant mesothelioma of epithelial type with tubulopapillary morphology (HE staining, magn. 200x).

Our patient presented also with long-term hydrocele. This shows a possible role of combined risk factors in the development of the disease. The median age of diagnosed patients is 54.1 years, but age dispersion is wide - from 12 to 76 years (10). Almost all patients are not diagnosed correctly until they are operated for other cause. The most common pre-operative diagnosis is hydrocele ( $49.5 \%$ of cases), followed by testicular tumour (36.6 \%) and inguinal hernia (5.9\%). Other less frequent diagnoses are epididymitis, spermatocele, testicular torsion, and post-traumatic testicular lesion (3). Patients consult doctors usually with hydrocele (40\%), scrotal mass (33\%), scrotal pain (9\%), inguinal mass (3\%), or epididymitis (3\%) (8). Histological examination of tumours reveals purely an epithelioid type of mesothelioma in $83 \%$ of cases showing a mixture of papillary, tubular, and solid patterns, and mixed sarcomatoid/epithelioid pattern in $17 \%$. Calretinin and EMA are always expressed; thrombomodulin is expressed in $89 \%$ of cases and CK7 in only $83 \%$. Tests for CK20 and CEA antigens are always negative. All mentioned antigens are similar to antigens of pleural malignant mesothelioma (11). Although the primary diagnosis is usually incorrect, an ultrasonographic examination can help with the diagnosis. The usual picture of appropriate hydrocele can be accompanied with that of tissue fronds with a hypoechoic centre surrounded by a hyperechoic rim which extends to hydrocele with multiple nodular masses of increased echogenicity arising from the scrotal wall or local nodular thickening of tunica vaginalis testis. (12) CT and MRI are suitable for staging the disease (13). A proportion of $33.7 \%$ of tumours are locally invasive when diagnosed. In case of hydrocele wall resection, $35.7 \%$ of tumours develop local recurrence, compared with only $10.5 \%$ in case of scrotal and $11.5 \%$ in case of inguinal orchiectomy. A proportion of $52.5 \%$ of patients develop local recurrence within 12 months, $83.9 \%$ of them with systemic dissemination, and $92.9 \%$ of patients are diagnosed with recurrence of the disease within 5 years. Metastases are most often found in lymph nodes (13.8\%), lung (9.7 \%), and liver (4.2 \%). (13) Mean disease-specific survival is 


\section{4-576}

36 months in patients with systemic treatment and 26 months without therapy (14). The survival of our patient was better; he was 20 months after resection of local recurrence with no evidence of the disease and with overall survival of 44 months after the primary treatment. Radiotherapy shows to be more efficient than chemotherapy with no benefit of combined therapy, and better results in young patients (13).

\section{Learning points}

- Extremely rare tumour,

- Patients usually undergo surgery for hydrocele; correct diagnosis set secondarily,

- Orchiectomy is indicated.

\section{References}

1. Scott B, Mukherjee S, Lake RA, Robinson BWS. Textbook of lung cancer. London: Martin Dunitz 2000; 273-293.

2. Antman K, Hassan R, Eisner M, Ries LA, Edwards BK. Update on malignant mesothelioma. Oncology (Williston Park) 2005; 19: 1301-130 9.

3. Yen CH, Lee CT, Su CJ, Lo HC. Malignant mesothelioma of the tunica vaginalis testis: a malignancy associated with recurrent epididymitis? World J Surg Oncol 2012; 10:238.

4. http://www.eternit.at/9935.0.html

5. Barbera V, Rubino M. Papillary mesothelioma of the tunica vaginalis. Cancer 1957; 10: 183-189.
6. Alesawi AM, Levesque J, Fradet V. Malignant mesothelioma of the tunica vaginalis testis: comprehensive review of literature and case report. J Clin Urol 2013; doi:10.1177/2051415813511082

7. Mrinakova B, Ondrus D, Kajo K, Kunderlik M, Tkacova M, Ondrusova M. Paratesticular mesothelioma in young age. Case report. Klin Onkol 2012; 25: 290-293.

8. Peterson JT, Greenberg SD, Buffler PA. Non-asbestos-related malignant mesothelioma. Cancer 1984; 54: 951-960.

9. Gürdal M, Erol A. Malignant mesothelioma of tunica vaginalis testis associated with long-lasting hydrocele: could hydrocele be an etiological factor? Int Urol Nephrol 2001; 32: 687-689.

10. Bisceglia M, Dor DB, Carosi I, Vairo M, Pasquinelli G. Paratesticular mesothelioma. Report of a case with comprehensive review of literature. Adv Anat Pathol 2010; 17: 53-70.

11. Winstanley AM, Landon G, Berney D, Minhas S, Fisher C, Parkinson MC. The Immunohistochemical profile of malignant mesotheliomas of the tunica vaginalis: a study of 20 cases. Am J Surg Pathol 2006; 30 (1): 1-6.

12. Mak CW, Cheng TC, Chuang SS, Wu RH, Chou CK, Chang JM. Malignant mesothelioma of the tunica vaginalis testis. Br J Radiol 2004; 77: 780-781.

13. Plas E, Riedl CR, Pflüger H. Malignant mesothelioma of the tunica vaginalis testis: review of the literature and assessment of prognostic parameters. Cancer 1998; 83: 2437-2446.

14. Spiess PE, Tuziak T, Kassouf W, Grossman HB, Czerniak B. Malignant mesothelioma of the tunica vaginalis. Urology 2005; 66: 397-401.

Received August 19, 2014. Accepted February 21, 2015. 